\title{
Planificación estratégica administrativa para pymes en tiempos post Covid. Caso de estudio de empresa textil para la toma de decisiones 2019-2020
}

\section{Strategic administrative planning for SMEs in post Covid times. Textile company case study for decision making 2019-2020}

Lourdes Rufina Parra Trelles

Instituto Superior Tecnológico Almirante Illingworth, Ecuador

Pablo Ricardo San Andrés Reyes

Universidad ECOTEC, Ecuador

Ivonne Elizabeth Paredes Chévez

Investigador Independiente, Ecuador

Autor para correspondencia: 1parra@aitec.edu.ec; psanandres@ecotec.edu.ec;

ivonnelizabeth.paredes@gmail.com

Fecha de recepción: 16 de septiembre de 2020 - Fecha de aceptación: 15 de noviembre de 2020

\section{Resumen}

Las Pymes en tiempos post covid necesitan de la planificación estratégica administrativa para mejorar la toma de decisiones e incremento de la rentabilidad. El objetivo del estudio es realizar el análisis integral de la planeación estratégica administrativa para la Pyme textil Amy's Factory Jeans, período 2016-2018, de tal manera que le permita fomentar la rentabilidad en tiempos post covid y mejorar la toma de decisiones 2019-2020. La metodología es descriptiva, bibliográfica y explicativa, además se ha aplicado un diseño no experimental de tipo transversal con muestreo no probabilístico dirigido y enfoque mixto, utilizando como herramientas de recolección de datos la entrevista semiestructurada a la gerencia, el grupo focal a miembros de la organización y el Cuadro de Mando Integral. Los principales resultados encontrados son: la empresa no cumplió los objetivos planteados en un $100 \%$ por la inejecución de las metas propuestas, debido a que las decisiones son tomadas de manera empírica sin la correcta utilización del plan estratégico.

Palabras claves: planificación estratégica; Pymes post covid; toma de decisiones; cuadro de mando integral; grupo focal.

\begin{abstract}
SMEs in post covid times need strategic administrative planning to improve decision-making and increase profitability. The objective of the study is to carry out a comprehensive analysis of the administrative strategic planning for the SME textile Amy's Factory Jeans, period 2016-2018, that allows it to increase profitability in post-covid times and improve decision-making 2019-2020. The methodology is descriptive, bibliographic and explanatory, including a non-experimental cross-sectional study, a purposive sampling, and a mixed approach. It was also used a semi-
\end{abstract}


structured management interview, a focus group for members of the organization and the Balanced Scorecard as data collection tools. The main results found are: the Company didn't get the objectives plans by $100 \%$ due to the non- execution of the proposed goals, because the decisions are made empirically without the correct use of the strategic plan.

Keywords: strategic planning; post covid SMEs; decision making; balanced scorecard; focus group.

\section{Introducción}

Las Pymes nacen producto de la contingencia y se moldean gracias a la tecnología, generando mayor productividad y llevando los costos financieros con mejor planificación (Seguel y Navarro, 2007). La crisis económica causada por el Covid-19 hizo que los gobiernos de los países de América Latina y el Caribe desarrollen nuevas políticas públicas y planes de reactivación económica (Bonaglia, Nieto, y Vásquez, 2020).

Los autores anteriores recalcan la rapidez con que han actuado los países, manteniendo distanciamiento social para minimizar los contagios, también con políticas fiscales para proteger a las personas y su capacidad humana, productiva y financiera. Los países han adoptado enfoques diferentes para manejar los mercados laborales, ha aumentado el uso de internet, emprendimientos que se han adaptado a la pandemia, enfrentando nuevos desafíos para navegar con éxito en su crecimiento, como la comunicación, entretenimiento y compras en línea (Donthu y Gustafsson, 2020). Inclusive, procesos de gestión para empresas Pymes a través de la innovación y la tecnología, tuvieron que modificarse, así como los procesos empresariales, acorde a la necesidad del mercado.

Las medianas, pequeñas y micro empresas representan el $90 \%$ del sector empresarial (INEC, 2019). Adicionalmente, el 90\% de estas empresas fracasan antes de los cinco años, por la inadecuada planificación, a falta de plan de negocios, con objetivos claros y metas a corto, mediano y largo plazo y un análisis de riesgos (GEM Ecuador, 2018).

Los principios de la Administración Científica según Taylor son: a) el procedimiento científico reemplaza al método empírico; b) existe selección científica y capacitación a trabajadores; c) la colaboración en el trabajo para su ejecución; d) división de trabajo y responsabilidad entre gerencia y trabajadores. Mientras que Fayol se concentró en la organización formal y actividades de los gerentes, como la planeación, organización, dirección y control (Robbins y Coulter, 2018).

La planificación estratégica ha aumentado conforme al crecimiento de las empresas en los distintos mercados y sus cambios. El Cuadro de Mando Integral mejora la comunicación, capacidad de análisis y la toma de decisiones por parte de la empresa. Gibb y Scott, 1985; Kantis, 1996 citado por Maldonado y Erazo (2016), señalaban que las Pymes carecían de procedimientos formales de planeación estratégica.

La planificación estratégica permite coordinar acciones y diferentes criterios para resolver problemas y conflictos de intereses dentro de la organización para cumplir con los objetivos empresariales (Ogliastri, 2004). De hecho, en las Pymes familiares se requiere de una 
planificación estratégica, con adecuada delimitación de funciones entre agentes externos e internos (Acosta y Pérez, 2010).

Simonds, 1981; Bromwich, 1990 citado por Feijóo, Gago, y Urrutia (2003), establecen que una correcta planificación estratégica necesita de una contabilidad estratégica empresarial, que permita analizar la información financiera para el diseño y revisión de la estrategia considerando la competencia actual. La planificación estratégica utiliza herramientas como el Cuadro de Mando Integral o el modelo de negocio Canvas, para implementación de estrategias (Vásquez, Elorza, y Pinzón, 2016).

Hoque y James, 2000; Speckbacher, Bischof y Pfeiffer, 2003; Papalexandris, Ioannou y Pratascos, 2004 citado por Argüello y Quesada (2015), hacen énfasis en la utilización del Cuadro de Mando Integral en empresas pequeñas, para la implementación de estrategias y toma de decisiones. Osterwalder, Pigneur y Tucci, 2005 citado por Vásquez, Elorza, y Pinzón, (2016), indican que el modelo de negocios Canvas facilita visualizar, comunicar y compartir la lógica del negocio y la relación entre áreas que deben tomar decisiones.

Por otra parte, a pesar de los años, la empresa textil Amy's Factory Jeans, por sus actividades operativas no crece en función de los objetivos. El problema está en que la empresa toma decisiones de manera empírica, sin realizar análisis de los indicadores de gestión de la planificación estratégica, lo que conlleva a generar gastos operativos innecesarios, como el stock de inventarios causando pérdidas.

El objetivo del estudio es realizar el análisis integral de la planeación estratégica administrativa para la Pyme textil Amy's Factory Jeans, período 2016-2018 que le permita incrementar la rentabilidad en tiempos post covid y mejorar la toma de decisiones 2019-2020. La importancia de esta investigación radica en la necesidad de realizar un diagnóstico de cumplimiento de la planificación estratégica de la empresa textil, a través del análisis de datos de los estados financieros y una medición de sus índices para que se tomen mejores las decisiones en la institución y se eviten pérdidas.

\section{Metodología}

La metodología es descriptiva, bibliográfica y explicativa, en cuanto al método es de diseño no experimental de tipo transversal de muestreo no probabilístico dirigido y enfoque mixto, utilizando como herramientas de recolección de datos la entrevista semiestructurada a la gerencia, el grupo focal a miembros de la organización y el Cuadro de Mando Integral.

La investigación es descriptiva, porque describe los datos de la situación del problema actual de la empresa y con esa información determinar cómo influyen en la efectividad de la planeación estratégica para la correcta toma de decisiones. Además es bibliográfica porque se ha consultado a diversas fuentes para la investigación y es explicativa, ya que permitirá identificar las causas del por qué la planificación estratégica actual no logra cumplir las metas establecidas por la empresa textil. 
El estudio es de diseño no experimental de tipo transversal de muestreo no probabilístico dirigido, debido a que la información se recoge en un tiempo único y la entrevista es realizada a la gerente de la empresa y el grupo focal a integrantes claves del departamento de compras, producción, diseño y distribución, para conocer el compromiso del plan estratégico respecto a la toma de decisiones.

La entrevista es un modelo de integración dialéctica, una conversación fluida, recíproca e interpersonal, utilizada como técnica en la investigación social, donde se obtiene conocimiento de opiniones y sentimientos, lo que produce un acercamiento real al campo de estudio, donde la persona se siente a gusto, confiada y dispuesta a colaborar (Carballo, 2001).

En la entrevista dirigida a la gerencia se consultaron las siguientes inquietudes: 1) Acerca de la planificación estratégica: ¿fue socializada entre todos y designaron responsables para el seguimiento y control?; 2) ¿Se realizó un presupuesto económico para el plan estratégico?; 3) ¿Cómo considera la toma de decisiones en la organización?; 4) Desde su punto de vista, ¿La planificación estratégica ha contribuido con información para la toma de decisiones?; 5) ¿Las decisiones tomadas han solucionado problemas empresariales?; 6) ¿Cuáles son los problemas que requieren soluciones inmediatas en la organización?

El grupo focal es una técnica de recolección de datos que permite realizar una entrevista de manera grupal alrededor de una temática, privilegiando la profundidad alcanzada de la información y logrando la interacción del grupo. Desde la perspectiva constructivista contextual y realista, logra dar pertenencia al grupo, homogeneidad, interacción y generalización de resultados (Escobar y Bonilla, 2017).

De hecho, para el grupo focal, se realizaron las siguientes preguntas: 1) ¿Los objetivos estratégicos de la empresa, son claros para la implementación?; 2) ¿Cómo ha contribuido para ustedes la ejecución del plan estratégico?; 3) ¿Qué herramientas utilizan para el seguimiento y control del plan estratégico?; 4) ¿El plan estratégico es una herramienta de gestión que les proporciona información para la toma de decisiones?; 5) ¿Tienen algún aporte adicional que puedan agregar al encuentro de grupo focal?

El enfoque de la investigación es mixto debido a que se realiza el análisis de los estados financieros para medir las metas y con el Cuadro de Mando Integral se puede corroborar el grado de cumplimiento de metas junto con la toma de decisiones.

Adicionalmente, el Cuadro de Mando Integral o en inglés Balanced Scorecard creado en 1992 por Kaplan y Norton, mide el desempeño de todas las actividades de una empresa. Establece una relación de causa y efecto a través del enfoque: financiero, clientes, procesos internos y aprendizaje, contienen indicadores y medidas de desempeño.

Quesado, Guzmán y Rodríguez, 2012 citado por Maldonado y Erazo (2016), proponen el Cuadro de Mando Integral o la implementación de sistemas de mejora continua para la mejora de la planificación estratégica. 
El Cuadro de Mando Integral es un tablero de control que combina indicadores financieros y no financieros, recoge procesos operativos, aplica lógica causa-efecto, utiliza los recursos materiales y humanos, cada empresa depende del sector al que pertenece y las estrategias que utilice y tiene como ventaja comparar los planes con los resultados para la revaluación y reajuste de estrategias (Dávila, 1999).

Ganesh, Mohapatra y Nagarajan, 2014 citado por Argüello y Quesada (2015), señalan una adaptación del principio básico del Cuadro de Mando Integral: si la empresa tiene el personal adecuado, hacen las cosas correctas, los clientes estarán satisfechos, se incrementará el negocio y obtendrá rentabilidad.

Para la medición de los objetivos estratégicos se cuenta con los indicadores de gestión (KPI) como son: indicadores financieros; de clientes; de procesos internos y de innovaciónaprendizaje (Espinosa, 2019).

El autor anterior, menciona a los indicadores financieros: rentabilidad económica, beneficio neto, deuda a corto plazo, deuda a largo plazo, deuda total, capital circulante, beneficio por empleado y dividendo por acción. Adicionalmente, entre los indicadores de clientes se tiene: número de reclamaciones y visitas a clientes, cuotas de mercado, ventas por cliente, cuota de fidelidad y de satisfacción, clientes perdidos, beneficio por clientes.

Entre los indicadores de procesos internos están: cumplimiento de auditorías, evaluación de desperdicios, tiempos muertos, índice de mantenimiento, coste de transporte, producción neta, tiempo de ciclo de proceso. Finalmente, entre los indicadores de aprendizaje se cuenta con: índices de calidad de formación, coste de la formación, satisfacción de empleados, índice de incentivos, tasa de abandonos e índices de comunicación.

\section{Estado del Arte: Planificación estratégica administrativa y toma de decisiones}

La planificación estratégica consiste en un proceso sistemático que permite el análisis y evaluación para mejorar la toma de decisiones (García, y otros, 2017). En las empresas ecuatorianas, el $95 \%$ consideran a la planificación estratégica como herramienta gerencial y aun así el $61 \%$ no lo ponen en práctica (Cho, 2017). Un error en el diseño o en la propia planificación, puede llevar a la toma de decisiones equivocadas para el negocio (Rodríguez y Pedraja, 2007).

La planeación estratégica ha evolucionado con los años desde 1960 hasta 1990. De hecho, en 1960 existen proyecciones a largo plazo, presupuestos a cinco años, planes operativos, estrategias de crecimiento, proyección tecnológica, planeación de fuerza laboral, matriz producto-mercado (David, 2003). Adicionalmente, el autor anterior muestra que durante 1970 se cuenta con: estrategias explícitas, divisiones, planeación, simulación de alternativas, aprobación de riesgo político, proyección social, evolución de impacto ambiental y análisis de sensibilidad y riesgo.

No obstante, en 1980 se cuenta con: la alta gerencia a cargo del plan estratégico, elaboración y marcha del plan estratégico, liderazgo de alta gerencia, inversiones en tecnología y 
objetivos empresariales, bases de datos. Finalmente, en 1990 se enfocan los mercados mundiales, la importancia de factores externos, las tecnologías de información, cálculos de riesgo, renovación del conocimiento, ventajas competitivas, benchmarking, inteligencia emocional, el mejoramiento continuo y cuadro de mando integral.

La contabilidad estratégica como parte de un modelo estratégico empresarial, permite obtener la información necesaria a través de los sistemas de información para la toma de decisiones (Feijóo, Gago, y Urrutia, 2003). La planificación estratégica tiene etapas: formulación, implementación y evaluación (David, 2003). Además, requiere de un presupuesto operativo por empresa o unidades de negocio, interactuando con las áreas funcionales, luego se negocia y se aprueba, es una experiencia que puede tomar tres o más años, constituyéndose un aprendizaje institucional (Ogliastri, 2004).

No obstante, la formulación de estrategias competitivas, permiten alcanzar ventajas competitivas a nivel empresarial y poder gestionar los negocios y actuar con sinergia (Acosta y Pérez, 2010). Las herramientas de control que contienen lineamientos e indicadores para saber si una empresa cumple metas a través de sus estrategias entre ellas: Dirección por objetivos, dirección por políticas (Sainz, 2012). El autor anterior recalca que la dirección por objetivos: implica medir los resultados y compararlos con los objetivos fijados; y la dirección por políticas: se concentran en los procesos, análisis de causas que provocan los resultados.

Por otro lado, la toma de decisiones consiste seleccionar un curso de acción entre las alternativas a través de un plan (Weihrich, Cannice, y Koontz, 2017). Las decisiones se pueden clasificar: a) según la posición jerárquica: estratégicas, tácticas y operativas; b) según el método: programadas y no programadas; c) sintética: estructuradas, semiestructuradas y no estructuradas (Castillo, Rodríguez, y Osma, 2017).

Respecto a las decisiones de la posición jerárquica: las decisiones estratégicas son estructuradas y tomadas por los más altos funcionarios, mientras las tácticas son tomadas por los ejecutivos intermedios en lapsos de corto tiempo y las operativas por los directores inferiores como supervisores, en actividades rutinarias (Castillo, Rodríguez, y Osma, 2017).

Las decisiones según el método se refieren a: las programadas en referencia a un procedimiento o criterio que no se vuelve a repetir, mientras las no programadas son para sucesos inesperados y requieren de mucho juicio y criterio. En referencia a las decisiones sintéticas: las estructuradas son las programadas y utilizan métodos matemáticos y reglas de decisión para su diseño y elección; las semi estructuradas usan modelos de algoritmos una vez identificado el problema; y las no estructuradas son las que no se pueden usar modelos matemáticos ni reglas porque son no programadas.

Por otra parte, las Pymes necesitan incorporar los valores, misión, visión y objetivos para la continuidad empresarial, inclusive las Pymes familiares necesitan de una planificación estratégica, que les permita además responder a las oportunidades favorables con compromiso familiar (Acosta y Pérez, 2010). 
Adicionalmente, el autor anterior enfatiza que la Pyme familiar debe seguir pasos para tener una planificación estratégica empresarial que le permita tomar decisiones adecuadas: a) Realizar un análisis del estado actual de la organización; b) análisis financiero; c) análisis de mercado; d) conocer las metas actuales; e) seleccionar estrategias; f) evaluar los intereses de la familia.

Shank, 1989; Shank y Govindarajan, 1993 citado por Feijóo, Gago, y Urrutia (2003), indican la gestión empresarial como un ciclo, desde la formulación de estrategias, comunicarlas en la organización, implementar las estrategias y luego realizar controles para verificación del cumplimiento de objetivos. Es por ello que la planificación estratégica puesta en marcha, con una correcta aplicación permite fomentar nuevas estrategias e impulsa a tomar mejores decisiones, principalmente para las Pymes y también las familiares.

\section{Pymes en tiempos post covid}

La sociedad ha sido afectada por pandemias en el pasado, sin embargo, Jaworski, Kohli y Sahay, 2000; Vargo y Lucsh, 2011 citado por Donthu y Gustafsson (2020), indican que el Covid19 ha demostrado que los mercados son dinámicos y se mueven rápidamente, un ecosistema dinámico que genera valor. Las Pymes y mipymes son el motor para activar la economía en tiempos post covid, dado el aporte que realizan a la producción, al empleo y expansión de nuevos mercados a nivel local e internacional.

OCDE, CEPAL, CIAT, BID, 2019 citado por Bonaglia, Nieto, y Vásquez (2020), indican que se necesitan estrategias nacionales de desarrollo para salir de la crisis del Covid-19 en los sectores, a pesar de que el PIB se contraerá como resultado de las repercusiones económicas de la pandemia, por otro lado, la tecnología representa un papel elemental en el proceso de recuperación como una oportunidad.

Según OCDE y CEPAL, 2012 citado por Ron y Sacoto (2017), las Pymes en Latinoamérica representan el 99\% de empresas y generan empleo aproximadamente del $67 \%$. Además, tienen estructuras organizacionales pequeñas lo que les permite adaptarse más rápidamente a cambios en la economía (Ron y Sacoto, 2017).

En América Latina, las mipymes corresponden al 88\% de las empresas formales, aportan $27 \%$ al empleo y 3,2\% a la producción. De hecho, los programas de apoyo a las mipymes en relación al financiamiento, innovación, desarrollo de mercado, articulación productiva, asistencia técnica y formación, regímenes tributarios especiales han contribuido favorablemente (Dini, Stumpo, y Unión Europea, 2018).

Según CEPAL, 2011 citado por Carvajal, Granda, Burgos, y Hermida (2017), indican que Ecuador ocupa el 44\% de participación de empresas Pymes en América Latina y genera el 24\% de empleo. La acción gubernamental a través de la coordinación institucional en sistemas capitalistas, con un nuevo sistema socio institucional, son fundamentales para dar estabilidad a la acumulación del capital y al uso social del excedente para corregir diferencias en el orden estructural (Barajas, 2007). 
El estado a través de sus políticas y planes de gobierno debe alentar a las empresas Pymes a reactivarse con programas que fomenten el apoyo, el financiamiento a través de la banca pública y privada y beneficios tributarios que permitan a los empresarios estabilizarse en tiempos post covid.

En Ecuador, la inclusión financiera, el rol de la banca pública debe ser complementaria a la privada y a las cooperativas, concentrarse en sectores de mayor riesgo y estratégicos, como lo hace BANECUADOR, para el desarrollo rural; y Corporación Financiera Nacional (CFN) sus créditos son necesarios para el desarrollo y utilizar fondos de garantía para compartir riesgos (Neira, 2016).

La política fiscal mitiga los efectos económicos y sociales negativos de la pandemia del Covid-19 para la recuperación (Bonaglia, Nieto, y Vásquez, 2020). No obstante, un incentivo fiscal orientado a las Pymes, fue a través de la Ley Orgánica para la Reactivación de la Economía, Fortalecimiento de la Dolarización y Modernización de la Gestión Financiera del 2017, en donde las microempresas que inicien su actividad económica, están exentas del impuesto a la renta por 3 años, y para las micro y pequeñas y exportadoras rebajas de 3 puntos porcentuales en impuesto a la renta (Dini, Stumpo, y Unión Europea, 2018).

Mediante Decreto Ejecutivo 1021, para que los contribuyentes puedan tener fluidez y flujo económico afectados por el covid, dieron ampliación de plazos para pago del Impuesto al Valor Agregado (IVA) de los meses de abril, mayo y junio 2020 y para el pago del Impuesto a la Renta del 2019 (SRI, 2020).

Adicionalmente, mediante Decreto Ejecutivo 1114 se incluyeron modificaciones al reglamento de la Ley Orgánica de Simplificación y Progresividad Tributaria en donde las microempresas referidas en el catastro que expuso la Administración Tributaria, aquellas que mantuvieron un nivel de ventas menores a $\$ 300.000$ dólares y que poseen de 1 a 9 empleados registrados en el Instituto Ecuatoriano de Seguridad Social (IESS), deberán pagar el Impuesto al Valor Agregado e Impuesto a la Renta semestralmente, dejarán de ser Sujetos de Retención en la Fuente salvo excepciones y pagarán un impuesto a la renta fijo del $2 \%$ sobre el total de ventas menos retenciones recibidas (SRI, 2020).

De las medidas anteriormente expuestas, habría que analizar si realmente favorecen o no a los contribuyentes, por un lado, el Estado necesita recaudación de tributos y por otro habría que considerar si bajo el régimen anterior estas empresas podían bajar la base y pagar un impuesto a la renta menor y con las nuevas reglas pagarán un porcentaje fijo de Impuesto a la Renta. Los factores formales de las instituciones, son las políticas del gobierno en relación a la creación de empresas, con mecanismos de fomentos para la creación y actividad empresarial mientras que los factores informales con actitudes de la sociedad limitan la creación de empresas (Díaz, Urbano, y Hernández, 2005).

Los consumidores se adaptan rápidamente al mercado, como la tecnología digital a través de servicios y compras en línea y diversas plataformas que permiten conectar a las personas en todo el mundo. Por lo tanto, nace una oportunidad para que las empresas participen con sus 
estrategias y reevalúen sus objetivos y logren generar cambios para sus clientes y proveedores (Donthu y Gustafsson, 2020).

Los empresarios Pymes deben mantener una adecuada planificación estratégica, para no recaer en excesos de stock de inventarios, implementar estrategias acorde a su realidad económica, un manejo adecuado para mantener la fidelidad de los clientes e incrementar el número de clientes, aumentar los ingresos y reducir costos de producción y los gastos innecesarios.

El acceso a la tecnología y nuevas plataformas permiten rediseñar las estrategias del plan estratégico, se ha convertido en la vitrina no sólo como oportunidad de expansión a nivel nacional sino internacional, permitiendo formar redes de clientes gracias a la globalización y reactivación económica post covid. Lograr una armonización entre el plano político, económico, científico y tecnológico y cultural, que logre el desarrollo local, es parte del modelo endógeno, que se da por la innovación en el proceso productivo, utilización de economías externas y produce cambio de matriz productiva y crecimiento económico para los diversos sectores (Fernández, Borré, Bracho, y Morales, 2012).

\section{Resultados}

Los principales resultados en base a los objetivos planteados en el estudio son: la empresa no cumplió los objetivos planteados en un $100 \%$ por la inejecución de las metas propuestas, debido a que las decisiones son tomadas de manera empírica sin la correcta utilización del plan.

La empresa Pymes textil Amy's Factory Jeans, tiene 9 años en el mercado, sin mostrar un crecimiento ni rentabilidad conforme a las características y competencia en el medio. Ofrece prendas diseñadas y fabricadas en tela jean para hombres y mujeres con precios de venta entre \$15.00 y \$30.00 dólares. Los productos pueden adquirirse en Cuenca, Guayaquil, Quito y Esmeraldas, también dispone de la modalidad catálogo físico y digital.

La gerencia quien se relaciona con todas las actividades de la organización, respecto a las inquietudes a través de la entrevista se obtuvieron los siguientes resultados:

1) La planificación estratégica fue realizada sin considerar al personal para el levantamiento de información, cuando se presentó el plan no se designaron responsables; 2) No se consideraron recursos económicos en el presupuesto anual; 3) En algunas ocasiones se han tomado decisiones de forma imprevista, de manera empírica, sin previo análisis; 4) El plan estratégico le ha contribuido con información para la toma de ciertas decisiones; 5) Las soluciones que se han tomado de forma imprevista o empírica no han solucionado los problemas; 6) Los problemas que requieren soluciones inmediatas en la organización son los existentes por la competencia agresiva respecto al precio y copia de diseños en el mercado informal del sector textil, disminuyen las ventas, los consumidores tienden a comprar donde existen precios bajos sin considerar calidad, lo que nos causa pérdidas y costos elevados afectando directamente a la empresa. 
A través del grupo focal dirigido a integrantes claves del departamento de compras, producción, diseño y distribución se obtuvieron las siguientes respuestas: 1) A pesar de conocer la visión, metas y objetivos de la empresa, no comprenden como llevar a la acción la aplicación del plan estratégico; 2) La contribución a la ejecución del plan estratégico ha sido mínimo, no tienen un líder encargado del mismo para la retroalimentación; 3) Para el seguimiento y control del plan estratégico no se utiliza ninguna medida de evaluación, sólo la verificación de datos en los estados financieros; 4) La información brindada por el plan estratégico consideran de utilidad para la toma de decisiones; 5) Reconocen estar vinculados al proceso desde la planificación hasta la ejecución para el desempeño eficiente y eficaz.

Posteriormente, en la revisión de la planificación estratégica 2016-2018, se verificó que la misión, visión, objetivos y planes se encontraron alineados con la estrategia del negocio en relación a calidad-precio. La información proporcionada en la planificación estratégica no es completa, no incluyen tampoco a responsables del seguimiento y control. Hubo estrategias no ejecutadas, otras en espera de que la empresa tenga liquidez, los estados financieros reflejaron gastos, afectando la toma de decisiones.

El Cuadro de Mando Integral tuvo los siguientes resultados:

En la perspectiva clientes, de acuerdo con la meta de lograr el $80 \%$ de satisfacción de clientes, está en rango de peligro, a causa de no realizar las encuestas, hacer seguimiento interno para medir grado de satisfacción y aceptación de productos, este indicador es importante para la toma de decisiones. De hecho, la estrategia no ha sido ejecutada efectivamente, ni considerada en el presupuesto anual de la empresa.

En la perspectiva financiera, para cumplir con el objetivo de incrementar las ventas en un $20 \%$ para el año 2018, el resultado fue $14 \%$ a pesar que las ventas han crecido en relación a años anteriores, está en rango de precaución porque no se cumplió en los tiempos estimados.

Respecto a la reducción de costos, con el objetivo de disminuirlos en un $15 \%$, el resultado fue $13.80 \%$, no llega a la meta de $15 \%$, sin embargo, se observa la reducción de costos debido al control sobre los desperdicios y fallas de las prendas al final del proceso productivo

Adicionalmente, de los datos de los estados financieros de la empresa de los años 2016 al 2018 se obtuvieron indicadores financieros: Margen neto de utilidad = Utilidad neta/Ventas Netas siendo 35\% para el año 2016; 34\% para el 2017 y 35\% para el año 2018.

Y el margen bruto de utilidad sobre ventas= Utilidad bruta en ventas/Ventas Netas; siendo 38\% para el año 2016; 37\% para el año 2017 y 37\% para el año 2018, lo que evidencia el uso eficiente de recursos de costos de producción, de todas formas se requiere enfoque en el tema de los desperdicios.

En la perspectiva interna, en cuanto al desarrollo de nuevos productos, se ha establecido diseñar 10 nuevos modelos por año, obteniendo como resultado apenas el $60 \%$ en los tres últimos años, lo que influye que sin nuevos diseños podría ser la razón por la que no llegó a la meta del incremento en ventas del $20 \%$. 
En la perspectiva de aprendizaje y crecimiento, la capacitación del recurso humano es importante, para que todas las actividades de la empresa funcionen adecuadamente, a pesar de que exista un plan anual de capacitaciones, no se presupuestó el costo, de hecho, sólo el $67 \%$ reciben capacitación mientras que el 33\% está en la espera de asignación de recursos para ello.

Es necesario, realizar un seguimiento y control sobre el cumplimiento de objetivos estratégicos para medir las metas a través de los indicadores e ir reformulando las acciones y las estrategias conforme a las necesidades organizacionales.

Además en el plan estratégico actual se concluye que la gerencia carece de información confiable para la toma de decisiones, los datos reales están lejos de lo establecido, la gestión administrativa no se guía por la planificación, las decisiones fueron tomadas sin considerar los indicadores lo que generó más costos, principalmente no hubo seguimiento ni control.

Entre los principales resultados se tiene que la empresa no cumplió los objetivos planteados en un $100 \%$ por la inejecución de las metas propuestas, debido a que las decisiones son tomadas de manera empírica sin la correcta utilización del plan.

Para que las decisiones de la empresa Amy’s Factory Jeans sean las adecuadas para el año 2019 y 2020 deben guiarse por el plan estratégico, realizar controles de seguimiento y rediseñar las estrategias conforme a las necesidades, así como la acción de estrategias para el cumplimiento de los objetivos, medición de las metas con los indicadores, tomándolos en consideración para el análisis de la toma de decisiones.

Un aspecto importante a considerar es que la dirección revise periódicamente, el cumplimiento de los objetivos estratégicos y sus planes de acción, inclusive Pymes con certificación poseen estrategias documentadas y procesos formales (Seguel y Navarro, 2007).

En ese sentido, la empresa ha tomado nuevos planes de acción para las decisiones del año 2019 y 2020 como son: implementación de una plataforma digital que mida la satisfacción del cliente, realización de nuevos diseños conforme a las necesidades del mercado, así como también el seguimiento y control de los desperdicios, rediseños de los procesos de forma integral sin perder la calidad y capacitaciones permanentes a todo el personal de la empresa. 


\section{Figura 1}

Cuadro de Mando Integral de la Empresa textil Amy's Factory Jeans

\begin{tabular}{|c|c|c|c|c|c|}
\hline $\begin{array}{l}\text { Iniciativas o plan de } \\
\text { acción }\end{array}$ & $\begin{array}{c}* * \\
\text { Implementación } \\
\text { de una } \\
\text { plataforma } \\
\text { digital que mida } \\
\text { la satisfacción } \\
\text { del cliente } \\
\end{array}$ & $\begin{array}{c}\text { *Nuevos } \\
\text { diseños } \\
\text { conforme a } \\
\text { las } \\
\text { necesidades } \\
\text { del mercado, } \\
\text { venta en línea } \\
\end{array}$ & $\begin{array}{l}* \text { Seguimiento } \\
\text { y control de los } \\
\text { desperdicios }\end{array}$ & $\begin{array}{c}* \text { Hacer } \\
\text { rediseños de } \\
\text { procesos de } \\
\text { manera } \\
\text { integral sin } \\
\text { perder calidad }\end{array}$ & $\begin{array}{l}\text { * Capacitaciones al } \\
\text { personal. }\end{array}$ \\
\hline \multirow{3}{*}{$\begin{array}{l}\text { RANGOS } \\
\text { DE } \\
\text { ALERTA }\end{array}$} & $20 \%$ & $10 \%$ & $10 \%$ & $10 \%$ & $30 \%$ \\
\hline & $20 \%<\mathrm{I}<99 \%$ & $10 \%<\mathrm{I}<15 \%$ & $10 \%<\mathrm{I}<20 \%$ & $10 \%<\mathrm{I}<99 \%$ & $31 \%<\mathrm{I}<99 \%$ \\
\hline & $80 \%$ & $20 \%$ & $15 \%$ & $100 \%$ & $100 \%$ \\
\hline VALOR ACTUAL & $0 \%$ & $14 \%$ & $13.80 \%$ & $60 \%$ & $67 \%$ \\
\hline 2018 & $0 \%$ & $5 \%$ & $3.40 \%$ & $20 \%$ & $17 \%$ \\
\hline 2017 & $0 \%$ & $4 \%$ & $4.00 \%$ & $17 \%$ & $33 \%$ \\
\hline 2016 & $0 \%$ & $4 \%$ & $6.40 \%$ & $23 \%$ & $17 \%$ \\
\hline Meta & $\begin{array}{l}\quad * 80 \% \text { de los } \\
\text { clientes } \\
\text { satisfechos }\end{array}$ & $\begin{array}{l}\quad * \text { Aumentar } \\
\text { las ventas } \\
\text { netas en un } 20 \\
\% \text { hasta el } \\
2018\end{array}$ & $\begin{array}{l}\text { *Reducir los } \\
\text { costos de } \\
\text { producción en } \\
\text { un } 15 \% \text { para el } \\
2018\end{array}$ & $\begin{array}{l}\quad * 10 \\
\text { productos } \\
\text { nuevos } \\
\text { diseñados } \\
\text { anualmente }\end{array}$ & $\begin{array}{l}* 100 \% \text { del } \\
\text { personal capacitado } \\
\text { al final del año } 2018\end{array}$ \\
\hline Indicadores & $\begin{array}{l}\text { *índice de } \\
\text { satisfacción del } \\
\text { cliente }\end{array}$ & $\begin{array}{l}* \% \text { de } \\
\text { ventas }\end{array}$ & $\begin{array}{l}\text { *Índice de } \\
\text { costos }\end{array}$ & $\begin{array}{l}\text { *\% de ventas } \\
\text { por nuevos } \\
\text { productos }\end{array}$ & $\begin{array}{l}* \% \text { de personal } \\
\text { capacitado. }\end{array}$ \\
\hline $\begin{array}{r}\text { Objetivos } \\
\text { estratégicos }\end{array}$ & $\begin{array}{l}\text { *Afianzar la } \\
\text { fidelidad de los } \\
\text { clientes hacia la } \\
\text { empresa }\end{array}$ & $\begin{array}{l}\text { *Aumentar } \\
\text { las ventas }\end{array}$ & $\begin{array}{l}\text { *Reducir los } \\
\text { costos }\end{array}$ & $\begin{array}{l}\text { Desarrollar } \\
\text { nuevos } \\
\text { productos en } \\
\text { líneas } \\
\text { económicas }\end{array}$ & $\begin{array}{l}\text { *Contar con } \\
\text { personal altamente } \\
\text { calificado en el } \\
\text { desarrollo de } \\
\text { productos. }\end{array}$ \\
\hline Perspectiva & Clientes & Financiera & & $\begin{array}{c}\text { Proceso de } \\
\text { Administración } \\
\text { de Operaciones }\end{array}$ & $\begin{array}{l}\text { Perspectiva de } \\
\text { aprendizaje y } \\
\text { crecimiento } \\
\text { Capital } \\
\text { organizacional } \\
\text { (liderazgo, y trabajo } \\
\text { en equipo), clima } \\
\text { laboral. }\end{array}$ \\
\hline
\end{tabular}

\section{Conclusiones}

Las Pymes en tiempos post covid, necesitan de la planificación estratégica administrativa para mejorar la toma de decisiones e incremento de la rentabilidad. 
Además, las Pymes tienen capacidad de fomentar empleo, cubrir demandas locales, con su estructura pequeña se adapta a fluctuaciones económicas y requieren de instituciones públicas y privadas para el desarrollo del país (Zambrano, San Andrés, y Paredes, 2019).

En la entrevista semiestructurada a la gerencia de la empresa Amy's Factory Jeans, se destacó que las decisiones en su mayoría fueron tomadas de forma empírica y sin considerar el plan estratégico, debido a la competencia desleal, copia de diseños y baja de precios en el mercado informal.

El grupo focal a los mandos medios del departamento de compras, producción, diseño y distribución ayudó a identificar que no conocen la forma para llevar a cabo las acciones del plan estratégico, y que hace falta una medida de valoración, además de verificación en estados financieros.

El Cuadro de Mando Integral, permite mediante indicadores, el cumplimiento de objetivos y estrategias para tomar mejores decisiones y obtener resultados favorables para la empresa. Del cual, se obtuvo que: La empresa Amy's Factory Jeans toma decisiones de manera empírica, sin realizar análisis de los indicadores de gestión de la planificación estratégica, lo que conlleva a generar gastos operativos innecesarios, como el stock de inventarios causando pérdidas. De hecho, la empresa no tiene responsable del seguimiento y control del plan estratégico, existen informes internos por los encargados del área, son revisados por la gerencia sin recurrir a los mandos medios.

La empresa no cumplió con el $100 \%$ de los objetivos, tuvo metas que no se ejecutaron, por lo que no se tomó en consideración el plan estratégico a la hora de tomar decisiones, debido a que la mayoría la hacen sin previo análisis, por el factor tiempo, sin embargo, le ocasionó excesivos gastos y pérdidas.

La planificación estratégica influye en la toma de decisiones para la efectividad de la empresa, utilizando óptimos recursos, por lo que la empresa, para la toma de decisiones 2019 y 2020, tomará en consideración los indicadores para el cumplimiento de las metas, acciones de estrategias rediseñadas conforme a las necesidades organizacionales y cumplimiento de los objetivos empresariales con mayor énfasis.

Las empresas Pymes en tiempos post covid para que puedan tomar mejores decisiones, necesitan identificar sus problemas, las falencias, realizar un cuadro de mando integral, rediseñar sus acciones y estrategias, establecer nuevas metas, utilizando los indicadores para la medición de las mismas y puedan cumplir con el logro de los objetivos de la empresa, alineados a la realidad económica y social.

No obstante, con la alineación de lo político, económico, científico y tecnológico, no sólo se logra el desarrollo local, sino la expansión internacional de las Pymes en situaciones post covid, con un modelo en donde accione lo público y lo privado y analicen las planificaciones estratégicas. 
Finalmente, la planificación estratégica administrativa se apoya en herramientas como el Cuadro de Mando Integral o el modelo de negocio Canvas, esperamos que en futuras investigaciones se puedan fusionar ambos diseños para su aplicación empresarial.

\section{Referencias bibliográficas}

Acosta, A., y Pérez, A. (2010). La planificación estratégica en las empresas familiares. CICAG, 7(2), 81-87. Obtenido de https://dialnet.unirioja.es/servlet/articulo?codigo=3706294

Argüello, E., y Quesada, C. (2015). Implementación del Cuadro de Mando Integral en pequeñas empresas: una revisión de literatura. Revista de Ciencias Económicas, 33(2), 79-122. doi:http://dx.doi.org/10.15517/rce.v33i2.22227

Barajas, G. (2007). Perspectiva de las micro y pequeñas empresas como factores de desarrollo económico de México. Centro de Estudios para el desarrollo nacional, 1-172. Obtenido de http://www.economia.unam.mx/profesor/barajas/perspec

Bonaglia, F., Nieto, S., y Vásquez, J. (2020). Una mirada al futuro post-COVID-19: hacia un nuevo pacto social en América Latina y el Caribe. Análisis Carolina(21), 1-15. doi:https://doi.org/10.33960/AC_21.2020

Carballo, R. (2001). La entrevista en la Investigación cualitativa. Pensamiento actual, 2(3), 1421. Obtenido de https://revistas.ucr.ac.cr/index.php/pensamientoactual/article/view/8017

Carvajal, C., Granda, L., Burgos, I., y Hermida, L. (2017). La importancia de las Pymes en Ecuador. Revista Observatorio de la Economía Latinoamericana. Obtenido de https://www.eumed.net/cursecon/ecolat/ec/2017/pymes-ecuador.html

Castillo, L., Rodríguez, D., y Osma, J. (2017). Modelo de simulación para evaluar las decisiones estratégicas de un comité local de AIESEC basadas en el Balanced Scorecard. Revista Ibérica de Sistemas y Tecnologías de Información(25). doi:10.17013/risti.25.82-97

Cho, B. (27 de Marzo de 2017). Planificación Estratégica en empresas familiares en Ecuador. Obtenido de Pymes Ecuador: http://brianchoec.weebly.com/crecimientoempresarial/planificacion-estrategica-empresas-familia-ecuador

David, F. (2003). Conceptos de Administración Estratégica. Mexico: Pearson Educación.

Dávila, A. (1999). El cuadro de mando integral. Revista de antiguos alumnos IESE, 34-42. Obtenido de http://www.geocities.ws/ivelisse_ocanto/BSC/Info5_archivos/Info_5.pdf

Díaz, J., Urbano, D., y Hernández, R. (2005). Teoría económica institucional y creación de empresas. Investigaciones europeas de dirección y economía de la empresa, 11(3), 209230. Obtenido de https://www.researchgate.net/publication/28200742_La_teoria_economica_institucional _el_enfoque_de_North_en_el_ambito_de_la_creacion_de_empresas

Dini, M., Stumpo, G., y Unión Europea. (2018). Mypymes en América Latina: un frágil desempeño y nuevos desafíos para las políticas de fomento. Santiago: Naciones Unidas. Obtenido de https://repositorio.cepal.org/bitstream/handle/11362/44148/1/S1800707_es.pdf 
Donthu, N., y Gustafsson, A. (2020). Effects of COVID-19 on business and research. Journal of Business Research, 117, 284-289. doi:https://doi.org/10.1016/j.jbusres.2020.06.008

Escobar, J., y Bonilla, F. (2017). Grupos focales: una guía conceptual y metodológica. Cuadernos Hispanoamericanos de Psicología, 9(1), 51-67. Obtenido de http://sacopsi.com/articulos/Grupo\%20focal\%20(2).pdf

Espinosa, R. (2019). Espinossa Consultores Marketing y Ventas. Obtenido de El cuadro de mando integral: conceptos y fases: https://robertoespinosa.es/2013/06/25/el-cuadro-demando-integral-concepto-y-fases

Feijóo, B., Gago, S., y Urrutia, I. (2003). El cuadro de mando integral en las Pymes: Un instrumento para su contabilidad estratégica. Partida doble, 145, 40-53. Obtenido de https://dialnet.unirioja.es/servlet/articulo?codigo=624853

Fernández, L., Borré, J., Bracho, N., y Morales, M. (2012). Empresa, conocimiento e innovación factores claves del modelo de desarrollo endógeno. Telos, 14(1), 121-150. Obtenido de https://www.redalyc.org/pdf/993/99322258008.pdf

García, J., Durán, S., Cardeño, E., Prieto, R., García, E., y Paz, A. (2017). Proceso de planificación estratégica: Etapas ejecutadas en pequeñas y medianas empresas para optimizar la competitividad. Revista Espacios, 38(52), 16. Obtenido de Recuperado de: https://www.revistaespacios.com/a17v38n52/a17v38n52p16.pdf

GEM Ecuador. (2018). Asociación Global de Investigación de Emprendimiento. London Business School. Reino Unido: Global Entrepreneurship Monitor. Obtenido de Recuperado de: https://www.gemconsortium.org/economy-profiles/ecuador

INEC. (30 de enero de 2019). Instituto Ecuatoriano de Estadísticas y Censos. doi:ECU-INECDECON-DIEE-2017-v1.5

Maldonado, G., y Erazo, M. (2016). El reto de la planificación estratégica en las Pymes. Revista publicando, 3(8), 335-344. Obtenido de https://revistapublicando.org/revista/index.php/crv/article/view/245/pdf_178

Neira, S. (2016). Inclusión financiera de las pymes en el Ecuador. CEPAL, 1-49. Obtenido de https://repositorio.cepal.org/handle/11362/40726

Ogliastri, E. (2004). Manual de planificación estratégica: Siete modelos para hacer un plan estratégico. INCAE Business School. Obtenido de https://www.academia.edu/35200113/MANUAL_DE_PLANIFICACION_ESTRAT\%C 3\%89GICA_Siete_modelos_para_hacer_un_plan_estrat\%C3\%A9gico

Robbins, S., y Coulter, M. (2018). Administración 13era edición. Pearson Educación en México.

Rodríguez, E., y Pedraja, L. (2007). Efecto e Implicaciones de las decisiones estratégicas en las instituciones universitarias. Interciencia, 32(9), 593-600. Obtenido de https://www.redalyc.org/pdf/339/33932904.pdf

Ron , R., y Sacoto, V. (2017). Las PYMES ecuatorianas: su impacto en el empleo como contribución al PIB PYMES al PIB total. Revista Espacios, 38(53), 15. Obtenido de https://www.revistaespacios.com/a17v38n53/a17v38n53p15.pdf

Sainz, J. (2012). El plan estratégico en la práctica (3era edición). Madrid: ESIC Editorial.

Seguel, N., y Navarro, C. (2007). El cuadro de mando integral y los sistemas normativos de gestión. Análisis para la pyme chilena. Revista Ingeniería Industrial, 6(1). Obtenido de http://revistas.ubiobio.cl/index.php/RI/article/view/119/3345 
SRI. (2020). Servicio de Rentas Internas. Obtenido de Administración Tributaria de Ecuador: https://www.sri.gob.ec

Vásquez, J., Elorza, M., y Pinzón, P. (2016). Balanced scorecard para emprendedores: desde el modelo canvas al cuadro de mando integral. Revista de la Facultad de Ciencias Económicas: Investigación y reflexión, 24(1), 37-47. doi:http://dx.doi.org/10.18359/rfce.1620

Weihrich, H., Cannice, M., y Koontz, H. (2017). Administración. Una perspectiva global empresarial y de innovación (15 edición). México: Mc Graw Hill/Interamericana editores S.A. de C.V.

Zambrano, R., San Andrés, P., y Paredes, I. (2019). Factores que inciden en las exportaciones de las Pymes del Ecuador. Período 2012-2016. Revista Espacios, 40(40), 4. Obtenido de http://www.revistaespacios.com/a19v40n40/19404004.html 